\title{
Robust Generation of Cardiomyocytes from Human iPS Cells Requires Precise Modulation of BMP and WNT Signaling
}

\author{
Asifiqbal Kadari • SubbaRao Mekala • Nicole Wagner • Daniela Malan • Jessica Köth • \\ Katharina Doll • Laura Stappert • Daniela Eckert • Michael Peitz • Jan Matthes • \\ Philipp Sasse • Stefan Herzig • Oliver Brüstle • Süleyman Ergün • Frank Edenhofer
}

Published online: 13 November 2014

(C) The Author(s) 2014. This article is published with open access at Springerlink.com

\begin{abstract}
Various strategies have been published enabling cardiomyocyte differentiation of human induced pluripotent stem (iPS) cells. However the complex nature of signaling pathways involved as well as line-to-line variability compromises the application of a particular protocol to robustly obtain cardiomyocytes from multiple iPS lines. Hence it is necessary to identify optimized protocols with alternative combinations of specific growth factors and small molecules to enhance the robustness of cardiac differentiation. Here we focus on systematic modulation of BMP and WNT signaling to enhance cardiac differentiation. Moreover, we improve the efficacy of
\end{abstract}

Electronic supplementary material The online version of this article (doi:10.1007/s12015-014-9564-6) contains supplementary material, which is available to authorized users.

A. Kadari $\cdot$ S. Mekala $\cdot$ D. Malan $\cdot$ P. Sasse $\cdot$ F. Edenhofer $(\triangle)$ Stem Cell and Regenerative Medicine Group, Institute of Anatomy and Cell Biology, University of Würzburg, 97070 Würzburg, Germany

e-mail: frank.edenhofer@uni-wuerzburg.de

K. Doll $・$ L. Stappert $\cdot$ D. Eckert $\cdot$ M. Peitz $\cdot$ O. Brüstle Institute of Reconstructive Neurobiologyy, University of Bonn-Life \& Brain Center, 53127 Bonn, Germany

A. Kadari $\cdot$ F. Edenhofer

Stem Cell Engineering Group, Institute of Reconstructive

Neurobiology, University of Bonn-Life \& Brain Center, 53127 Bonn, Germany

S. Mekala $\cdot$ N. Wagner $\cdot$ S. Ergün Institute of Anatomy and Cell Biology, University of Würzburg, 97070 Würzburg, Germany

D. Malan • P. Sasse

Institute of Physiology I, University of Bonn-Life \& Brain Center, 53127 Bonn, Germany

J. Köth $\cdot$ J. Matthes $\cdot$ S. Herzig

Department of Pharmacology, University of Cologne,

50931 Cologne, Germany cardiac differentiation by enrichment via lactate. Using our protocol we show efficient derivation of cardiomyocytes from multiple human iPS lines. In particular we demonstrate cardiomyocyte differentiation within 15 days with an efficiency of up to $95 \%$ as judged by flow cytometry staining against cardiac troponin T. Cardiomyocytes derived were functionally validated by alpha-actinin staining, transmission electron microscopy as well as electrophysiological analysis. We expect our protocol to provide a robust basis for scale-up production of functional iPS cell-derived cardiomyocytes that can be used for cell replacement therapy and disease modeling.

Keywords Human iPS cells · Cardiac differentiation - WNT signaling $\cdot$ BMP signaling $\cdot$ Lactate enrichment .

Disease modeling

\section{Introduction}

In spite of recent advances in medicine cardiovascular disorders remain a major cause of mortality in the world [1]. Supply with human cardiomyocytes is generally limited due to lack of donors as well as the restricted proliferation rate of adult cardiomyocytes. Thus, with respect to use human cardiomyocytes for regenerative therapies, drug toxicity studies as well as disease modeling alternative sources are highly desired. There have been many attempts in this direction using adult stem cells such as bone marrow derived stem cells (BMSCs) [2], mesenchymal stem cell (MSCs) [3], c-kit and isl-1 positive cardiac stem cells (CSCs) [4, 5]. However there is little evidence that BMSCs and MSCs differentiate into cardioymocytes after transplantation since positive effects observed using those cells are mainly due to angiogenesis and paracrine effects [6]. Although it has been shown that CSCs can be differentiated into all cardiovascular lineages in an animal model [7], in humans there have been rare studies due to lack of donors, limited in vitro amplification as well as complicated 
isolation procedures of the CSCs $[6,8]$. Embryonic stem (ES) cells hold great promise for providing an unlimited source of cardiac cells since ES cells self-renew indefinitely in cell culture and are able to differentiate into any somatic cell type [9]. However ethical considerations associated with the use of human embryos might represent a roadblock for clinical application [10]. Major breakthrough in this field came when Yamanaka and colleagues showed that overexpression of four transcription factors namely Oct-4, Sox2, Klf-4 and c-Myc were able to transform somatic cells into induced pluripotent stem cells (iPS) [11]. iPS technology allows generation of pluripotent stem cells from any somatic cells. Not only it overcomes ethical concerns associated with ES cells but also offers the potential of autologous transplantation since patientspecific cells can be used for cellular reprogramming [11].

Numerous protocols have been published reporting the derivation of cardiomyocyte-like cells from human ES and iPS cells. Induction of differentiation by co-culture with stroma cells has been demonstrated [12] as well as the use of embryoid body (EB) based differentiation paradigms [13, 14]. It is similar to embryonic development in some respect and cells from all three germ layers are formed during the course of differentiation. However EBs have complex microenvironments and for this reason signaling pathways are difficult to modulate explaining poor efficiency of cardiac differentiation [15]. Moreover, there is a significant line-to-line variability with respect to the method of reprogramming used and iPS quality resulting in up to 100 -fold differences in lineage specific gene expression amongst the lines treated with same protocols [16]. Such variability within a broad range of pluripotent cell lines greatly limits its application [17, 18]. Several approaches have been published utilizing monolayer culture of cells in a serum free condition having growth factors such as BMP4, Activin A, FGF2, VEGF in order to increase the efficiency while reducing the heterogeneity arising during EB based differentiation [19-21]. However, it has been shown that optimal concentrations of growth factors greatly vary among different iPS lines. A study by Kattman et al. as well as follow up report by Sa et al. systematically showed different requirements of Activin A and BMP4 concentration for efficient cardiomyocytes yield amongst different pluripotent cell lines $[22,23]$. Thus, robust and efficient cardiac differentiation requires the optimization of the protocol for each individual line, which makes it laborious [24].

Recent advances in cell signaling studies have shed light on detailed signaling pathways involved during cardiac differentiation. It has been shown that WNT signaling plays a critical role during cardiogenesis [25]. It has been suggested that during early embryonic differentiation WNT is required for mesodermal specification, however, later on cardiac specification is hampered by WNT signaling and thus inhibition of WNT signaling might be necessary for the formation of cardiomyocytes [26]. Recent studies have shown the use of WNT inhibiting small molecules for increasing the cardiomyocyte yield in the case of EB [27] as well as monolayer-based protocols [28, 29]. Moreover, two recent studies by Cao et al. have shown importance of ascorbic acid in increasing the cardiac differentiation efficiency by affecting MEK/ERK pathway. By combining the application of BMP4, CHIR99021 and ascorbic acid they were successful in isolation of cardiovascular cells from human pluripotent stem cells $[30,31]$. However, varied differentiation propensities of multiple pluripotent cell lines to a particular protocol require more alternative approaches. Hence, still it is highly desired to devise simple and efficient protocols to achieve high robustness and efficacy. Here we show improvement in cardiac differentiation efficiency using precise modulation of WNT and BMP signaling. We identify an optimal combination of concentrations of BMP4 and CHIR99021 and finally perform cardiomyocyte enrichment by lactate supplementation [32]. By that within 15 days we achieve generation of more than $90 \%$ cardiomyocytes as judged by cardiac Tropononin $T$ staining with unmatched low line-to-line variability.

\section{Materials and Methods}

Human iPSC Cultivation and Subsequent Cardiac Differentiation

Human iPSC were maintained on hESC-qualified Matrigel(TM) (BD Biosciences) coated plates in mTESR1 (STEMCELL Technologies) medium until they reached 80 to $90 \%$ confluency. Cardiac differentiation was induced by BMP4 (Life technologies) $(25 \mathrm{ng} / \mathrm{ml})$ and CHIR99021 (5 $\mu \mathrm{M})$ (Sigma-Aldrich) in RPMI1640 (Life technologies) medium containing B27 (Life technologies) and $2 \mathrm{mM}$ glutamine (Life technologies) and $50 \mu \mathrm{g} / \mathrm{ml}$ L-Ascorbic acid (Cell culture tested powder; SigmaAldrich) as a basal medium. After $24 \mathrm{~h}$ cells were kept in the same basal medium with CHIR $(5 \mu \mathrm{M})$ only for 18-36 h. Afterwards cells were kept in RPMI basal medium with B27 without insulin for $24 \mathrm{~h}$ then medium was replaced with similar basal medium having WNT inhibitor either $10 \mu \mathrm{M}$ of XAV939 (Sigma-Aldrich) or IWR1 (Sigma-Aldrich) for 5 days. Afterwards cells were kept 4 to 5 days in basal medium (B27 + insulin) followed by replacement with cardiac enrichment medium (RPMI 1640 without glucose (Life technologies) $+4 \mathrm{mM}$ sodium L-lactate [32] (Sigma-Aldrich). Cells were kept in enrichment medium for 4 to 5 days. After enrichment phase medium was switched back to basal medium (RPMI + B27 + glutamine).

\section{Cell Lines}

del-AR1034ZIMA 001 and fl-AR1034ZIMA 001 are lentiviral reprogramming derived iPS sister clones from human dermal fibroblasts (del: transgene excised, fl: transgene floxed [33]. Human iPS cell line (k-hiPS) [34] is a lentivirally derived iPS cell line from human keratinocytes kindly gifted by Dr. 
Stefan Liebau from University of Tübingen, Germany. Human iPS cell line iLB-C-50-s9 is a Sendai virus derived iPS cell line from human cord blood cells. Human iPS cell line iLB-C1$30 \mathrm{~m}-\mathrm{r} 12$ is a retroviral reprogramming derived iPS cell line from human dermal fibroblasts cells [35]. As WNT reporter cell line we used a neural stem cell line (I3 lt-NES) carrying the 7TGP WNT reporter construct developed by Fuerer \& Nusse (2010) [36] and obtained via Addgene (\#24305).

\section{Immunostaining}

For cardiomyocyte characterization, immunostaining was performed using anti-cTNT (Abcam; 1:100) and anti-alpha-actinin (Sigma-Aldrich; 1:200) as primary antibodies and Alexafluor488-cojugated anti-mouse IgG as a secondary antibody. Briefly, cells were washed with PBS (Life technologies), fixed with $4 \%$ PFA (Sigma-Aldrich) for 15 min and permeabilized in PBS containing $0.1 \%$ Triton X-100 (Sigma-Aldrich) and $5 \%$ FCS (Life techologies) for $30 \mathrm{~min}$. Cells were then incubated overnight with the primary antibodies. Next day, secondary antibody Alexafluor-488-cojugated anti-mouse IgG (1:1,000; Life technologies) were used to detect and visualize the primary antibodies. All antibodies were diluted in blocking solution. Similar protocol was also used for the staining using ISL1 (Biorbyt; 1:200) antibody. Micrographs were taken with an Axiovert 200 M microscope (Carl Zeiss).

\section{RT-PCR}

Total RNA was prepared with the NucleoSpin RNA kit (Macherey -Nagel) and treated with DNase (Thermo Scientific). RNA $(1 \mu \mathrm{g})$ was reverse transcribed into cDNA via Oligo (dT) with SuperScript III Reverse Transcriptase (Invitrogen). PCR was perfomed using Go Taq polymerase kit (Promega) with following conditions; $95{ }^{\circ} \mathrm{C}$ for $2 \mathrm{~min}$; followed by 34 cycles of $94{ }^{\circ} \mathrm{C}$ for $30 \mathrm{~s}, 60^{\circ} \mathrm{C}$ for $30 \mathrm{~s}$, and $72{ }^{\circ} \mathrm{C}$ for $45 \mathrm{~s}$; followed by a single cycle of $72{ }^{\circ} \mathrm{C}$ for $5 \mathrm{~min}$ using the primers [37] given below.

\begin{tabular}{|c|c|c|}
\hline Primers & Band size (bp) & $\operatorname{Tm}\left({ }^{0} \mathrm{C}\right)$ \\
\hline $\begin{array}{l}\text { Oct4-F: aac ctg gag ttt gtg cca ggg ttt } \\
\text { Oct4-R: tga act tca cct tcc ctc caa cca }\end{array}$ & 120 & 60 \\
\hline $\begin{array}{l}\text { Tbrachyury-F: tgt ccc agg tgg ctt aca gat gaa } \\
\text { Tbrachyury-R: ggt gtg cca aag ttg cca ata cac }\end{array}$ & 140 & 60 \\
\hline $\begin{array}{l}\text { ISL1-F: cac aag cgt ctc ggg att gtg ttt } \\
\text { ISL1-R: agt ggc aag tct tcc gac aa }\end{array}$ & 200 & 60 \\
\hline $\begin{array}{l}\text { Nkx2.5-F: gcg att atg cag cgt gca atg agt } \\
\text { Nkx2.5-R: aac ata aat acg ggt ggg tgc gtg }\end{array}$ & 220 & 60 \\
\hline $\begin{array}{l}\text { cTNT-F: ttc acc aaa gat ctg ctc ctc gct } \\
\text { cTNT-R: tta tta ctg gtg tgg agt ggg tgt gg }\end{array}$ & 160 & 60 \\
\hline $\begin{array}{l}\beta \text {-actin (BA)-F: ttt gaa tga tga gcc ttc gtc ccc } \\
\beta \text {-actin (BA)-R: ggt ctc aag tca gtg tac agg } \\
\text { taa gc }\end{array}$ & 130 & 60 \\
\hline
\end{tabular}

Flow Cytometry

$1 \times 10^{6}$ cells were trypsinized and fixed with $4 \%$ PFA for $10 \mathrm{~min}$. Cells were then washed with PBS, permeabilized in PBS containing $0.1 \%$ Triton X-100 and $5 \%$ FCS for 30 min and incubated for $2 \mathrm{~h}$ with cTNT antibody (Abcam; 1:100). No antibody was taken as a negative control. Cells were then washed once with PBS containing $0.1 \%$ Tween- 20 and resuspended in PBS containing $0.1 \%$ Triton-X 100 and $5 \%$ FCS and secondary antibody Alexafluor-488-cojugated antimouse $\operatorname{IgG}(1: 1,000$; Life technologies) for $1 \mathrm{~h}$ in dark. Finally, cells were washed again with PBS containing $0.1 \%$ Tween-20 and measured for FACS analysis. Analysis was performed by Flow Jo program.

\section{Whole-Cell Calcium Current Measurements}

Conventional whole-cell patch clamp recordings were performed at room temperature in bath solution containing (mM): $\mathrm{NaCl} 137, \mathrm{CsCl} 5.4, \mathrm{CaCl}_{2} 2, \mathrm{MgCl}_{2} 1$, glucose 10 , HEPES 10 (pH 7.4 with $\mathrm{NaOH})$. Borosilicate pipettes (2-3 $\mathrm{M} \Omega$ ) were filled with a solution containing (mM): $\mathrm{CsCl} 120$, $\mathrm{MgCl}_{2}$ 1, Mg-ATP 4, EGTA 10, HEPES 5 (pH 7.2 with $\mathrm{CsOH}$ ). Giga-Ohm seals were formed by gentle suction. Membrane capacitance was automatically displayed in the pClamp 10.2 software (Axon instruments). Cells were depolarized from a holding potential of $-80 \mathrm{mV}$ to $-40 \mathrm{mV}$ for $45 \mathrm{~ms}$ in order to inactivate sodium channels. This prepulse was followed by test voltages ranging from -40 to $+50 \mathrm{mV}$ in $10 \mathrm{mV}$ steps (pulse duration $150 \mathrm{~ms}$ ) with a $3 \mathrm{~s}$ interval between single pulses.

\section{Calcium Imaging}

Calcium imaging was performed in cell cultures loaded with $5 \mu \mathrm{M}$ of calcium indicator dye fluo-4AM (Life technologies). Briefly, the cells were cultivated in 6 well plates and incubated then with $1 \mathrm{ml}$ of loading dye solution containing fluo-4 AM at room temperature for $20 \mathrm{~min}$ in the dark. Movies were captured through the Keyence Microscope BZ-9000 (Keyence, Japan)

\section{Action Potential and Ramp Recordings}

Cardiomyocytes were enzymatically dissociated at day 30 and plated at low density on glass cover slips coated with fibronectin. Action potentials and voltage ramps from $-100 \mathrm{mV}$ to $60 \mathrm{mV}, 250 \mathrm{~ms}$ long were recorded on spontaneously beating cardiomyocytes with a EPC 10 amplifier (Heka Electronics) as previously described [38]. Electrode resistance was between 2.5 and $3.5 \mathrm{M} \Omega$, the pipette solution contained (in $\mathrm{mM}$ ): $50 \mathrm{KCl} ; 80 \mathrm{KAsparatate} ; 10$ EGTA; 10 Hepes; 3 MgATP; $1 \mathrm{MgCl}_{2}(\mathrm{pH} 7.2)$ and the extracellular solution 
contained (in $\mathrm{mM}$ ): $140 \mathrm{NaCl} ; 5.4 \mathrm{KCl} ; 1.8 \mathrm{CaCl}_{2} ; 1 \mathrm{MgCl}_{2}$; 10 Hepes; 10 Glucose (pH 7.4).

\section{Transmission Electron Microscopy}

Undifferentiated iPS cells or differentiated iPS cells at day 21 were fixed in $4.5 \%$ glutaraldehyde in $0.1 \mathrm{M}$ phosphate buffer pH 7.2 (PB). After washing with $0.1 \mathrm{M} \mathrm{PB}$, specimens were subsequently fixed for $1 \mathrm{~h}$ with $1 \%$ osmiumtetroxide in $\mathrm{PB}$ and washed with water. Specimens were then dehydrated in ascending concentrations of ethanol including en-bloc contrasting using $2 \%$ uranylacetate in $70 \%$ ethanol for $1 \mathrm{~h}$. Subsequently, they were embedded in Epon812 and used for preparation of ultrathin sections which were poststained with $2 \%$ uranylacetate and $0,2 \%$ lead citrate. The sections were observed using a LEO AB 912 transmission electron microscope (Zeiss NTS, Oberkochen, Germany)

\section{Results}

Defining Optimal Window of WNT Modulation to Achieve Efficient Myocardial Induction in Human iPSCs

Since cardiomyocyte differentiation critically depends on WNT signaling, our first step was to screen molecules with which one can tightly control WNT signaling in iPS cells and their progeny, both in an agonistic and antagonistic manner, respectively. In order to quantitatively assess the level of WNT signaling, we employed a WNT reporter cell line and assessed the functionality of candidate molecules to modulate WNT signaling. In order to identify potent WNT activator, we used CHIR99021 (designated CHIR hereafter) and BIO. We found that $5 \mu \mathrm{M}$ CHIR99021 (designated CHIR hereafter) strongly activates WNT signaling (data not shown) while BIO appeared toxic to the cells. To identify potent molecules with potent WNT inhibition, we screened previously described molecules XAV939, IWR1, KY02111 and WNT-C59. According to this analysis XAV939 and IWR1 showed strongest WNT inhibition without causing excessive cell death. Hence we decided to use CHIR as WNT activator and XAV939 or IWR1 as WNT inhibitor during the subsequent experiments. In order to achieve efficient cardiovascular induction we decided to formulate different combinations of growth factors and small molecules modulating important signaling pathways such as TGF $\beta$ (Activin A, BMP4) and FGF (FGF2) as well as WNT (CHIR, XAV939, IWR1) [15]. As a quick read out for cardiovascular induction we decided to check the expression of T-brachyury at day two (Fig. 1a) and Isll at day 5 (Fig. 1b) of differentiation. As an end-point analysis we monitored the capability of cultures to exhibit spontaneously beating patches in a semi-quantitative manner
(Suppl. Tabl. 1-3). Comprehensive quantification of cardiac differentiation was carried out by flow cytometry analysis using cTNT specific antibodies (Fig. 1c). According to these analyses we found that the combination of BMP4 $(25 \mathrm{ng} / \mathrm{ml})$ and CHIR $(5 \mu \mathrm{M})$ strongly enhanced expression of Tbrachyury whereas additional application of Activin A had no effect (Fig. 1a). Neither increasing the CHIR concentration nor extending the incubation period beyond $48 \mathrm{~h}$ had a beneficial effect (Suppl. Tabl. 1). Applying $25 \mathrm{ng} / \mathrm{ml}$ BMP4 turned out to be the optimal concentration since higher and lower concentrations, respectively, reduced the number of beating patches (Suppl. Tabl. 2). After an initial phase of WNT activiation the precise timing of WNT inhibition is critical. Our data shows that application of both WNT inhibitors, XAV939 or IWR1, between day 3 and 8 results in optimal cardiac differentiation (Suppl. Tabl. 3). After day 5 of differentiation cells showed strong expression of the early cardiac marker ISL1 in almost every cell (Fig. 1b). Using these optimized conditions we generated cardiomyocyte-like cells of up to $95 \%$ purity from human iPS line (iLB-C-50-s9) at day 12 of differentiation as judged by flow cytometry analysis using cTNT specific antibodies (Fig. 1c).

Lactate Based Cardiac Enrichment Strongly Reduces Line-to-Line Variability of Cardiomyocyte Differentiation

After optimization of cardiac differentiation using a standard iPS line, we checked the efficacy of the devised protocol on multiple iPS lines representing different origins of cells (fibroblasts, keratinocyte and cord blood cells) as well as methods of reprogramming (Retrovirus, Lentivirus and Sendai virus) to cover the full spectrum of state-of-the-art iPS technology (see details on iPS lines used in the materials section). Although our optimized protocol gave rise to a highly enriched population of beating cells with the standard iPS cell line (del-AR1034ZIMA 001), the outcome with the other iPS lines indeed varied substantially. In fact, we obtained yields of cTNT-positive cells ranging from 33 to $92 \%$ (Fig. 2a) demonstrating the high line-to-line variability using the basic standard protocol. In order to maximize purity of cardiomyocytes from different iPS lines to the same level, we decided to apply lactate based cardiac enrichment in the late phase of our protocol. As has been recently reported glucosedepleted, lactate-supplemented culture medium strongly selects for cardiomyocytes [32]. Since only cardiomyocytes can metabolize lactate for energy supply, other non-cardiac cells were expected to die out during this 4 days treatment resulting in higher purity of cardiomyocytes. In order to achieve this, we switched the medium at day 12 of cardiac differentiation to basal medium without glucose but supplemented with lactate. In fact when we applied lactate enrichment, we could obtain $95 \%$ pure cTNT-positive cells from the iPS line iLB-C30 -r12 which otherwise gave about $63 \%$ positive 
A

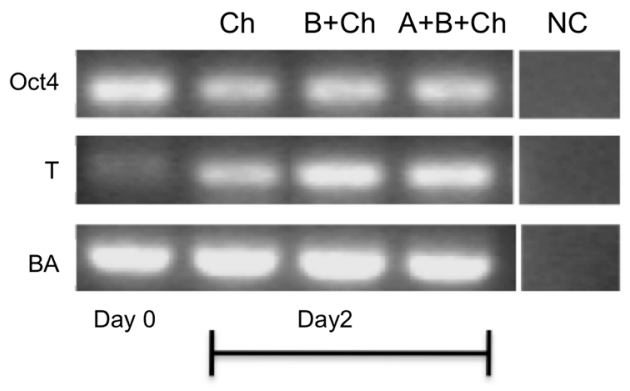

B

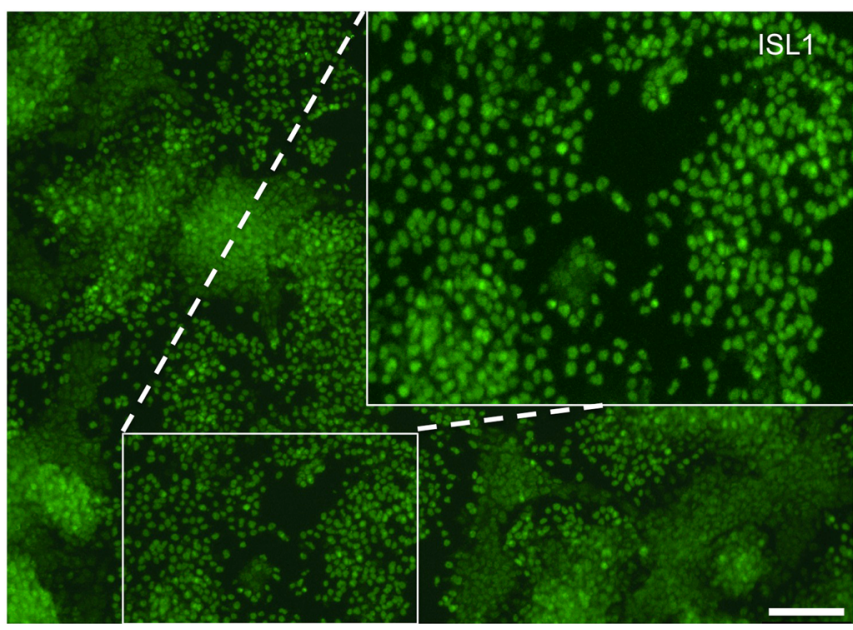

Fig. 1 Optimization of myocardial induction of human iPS line (iLB-C50-s9). a RT PCR analysis to assess the expression of T brachyury at day 2 of cardiac induction using different conditions, namely $\mathrm{Ch}, \mathrm{B}+\mathrm{Ch}$ and $\mathrm{A}+\mathrm{B}+\mathrm{Ch} \mathbf{b}$ Immunostaining using cardiac precursors maker ISL1 at day 5 of cardiac differentiation using small molecule combination (B + Ch). Scale bar: $100 \mu \mathrm{M}$ c Flow cytometry analysis of cardiac-specific

cardiomyocytes (Fig. 2a and b). Even the iPS line flAR1034ZIMA, carrying loxP-flanked reprogramming transgenes [35] and being strongly resistant towards cardiac differentiation, showed efficient enrichment from 34 to $74 \%$ cTNT-positive cells (Fig. 2a).

In conclusion our optimized protocol of cardiomyocyte differentiation from multiple human iPS lines represents a three phase protocol consisting of cardiac induction, specification and enrichment as outlined in Fig. 3a. During the induction phase iPS cells are treated with our formulation (BMP4 and CHIR) in a basal medium with insulin, which resulted in strong upregulation of the mesendodermal marker T-brachyury (Fig. 3b). Induction phase is followed by treatment with WNT inhibitors in basal medium devoid of insulin in order to achieve proper specification to cardiac mesoderm, which is confirmed by expression of early and late cardiac precursor markers ISL1 and Nkx2.5, respectively (Fig. 3b). Cells then further mature into beating cardiomyocytes expressing the mature cardiomyocyte marker cTNT (Fig. 3b). Once beating is observed cells are switched to basal medium with insulin followed by enrichment phase with basal medium devoid of glucose but supplemented with $4 \mathrm{mM}$ lactate for 4 days (Fig. 3a).

Characterization and Validation of Cardiomyocytes Derived from Human iPSCs

We performed a series of standard immunohistochemical and electrophysiological methods to assess the functionality of cardiomyocyte derived. Obtained cardiomyocytes showed strong cardiac specific alpha-actinin staining with typical striation pattern as well as cTNT staining (Fig. 3c). Moreover, patch-clamp recordings on single beating cardiomyocytes showed typical spontaneous action potentials (Fig. 3d), with atrial $(n=2)$ or ventricular $(n=4)$-like properties, as well as characteristic voltage-dependent inward and outward currents using voltage ramps (Fig. 3e). We recorded $\mathrm{Ca} 2+$ currents typical for L-type $\mathrm{Ca}^{2+}$ channels with a halfmaximum activation at $-13.69 \pm 0.97 \mathrm{mV}$, a maximum current density of $-11.55 \pm 1.6 \mathrm{pA} / \mathrm{pF}$ at $0 \mathrm{mV}$ and nearly complete 
A

\begin{tabular}{|l|l|l|}
\hline Human iPS lines & $\begin{array}{l}\text { \% cTNT w/o } \\
\text { enrichment }\end{array}$ & $\begin{array}{l}\text { \% cTNT with } \\
\text { enrichment }\end{array}$ \\
\hline iLB-C-50-s9 (Cord blood) & 92.5 & ND \\
\hline del-AR1034ZIMA 001(Fibroblast) & 82.4 & ND \\
\hline k-hiPS (Keratinocyte) & 42.4 & ND \\
\hline iLB-C1-30m-r12 (Fibroblast) & 62.8 & 95.5 \\
\hline fl-AR1034ZIMA 001 (Fibroblast) & 33.8 & 74.3 \\
\hline
\end{tabular}

B

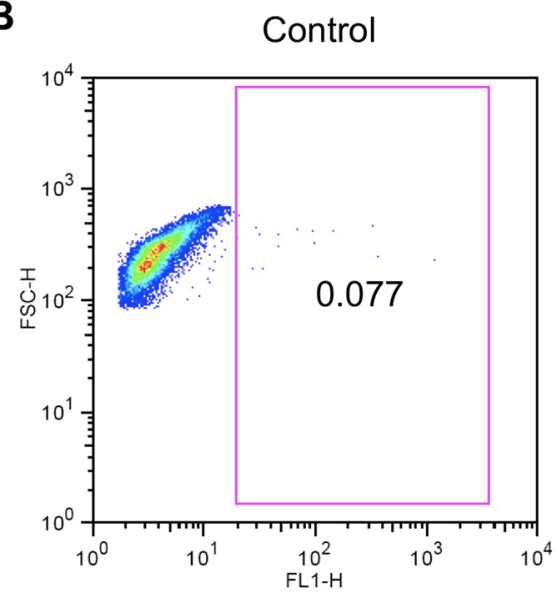

w/o lactate enrichment

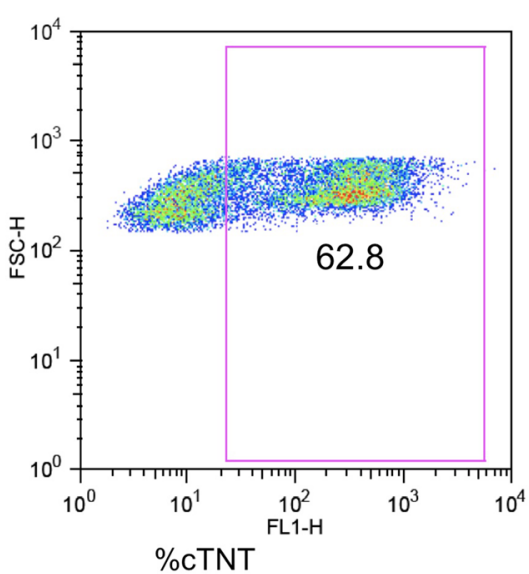

lactate enrichment

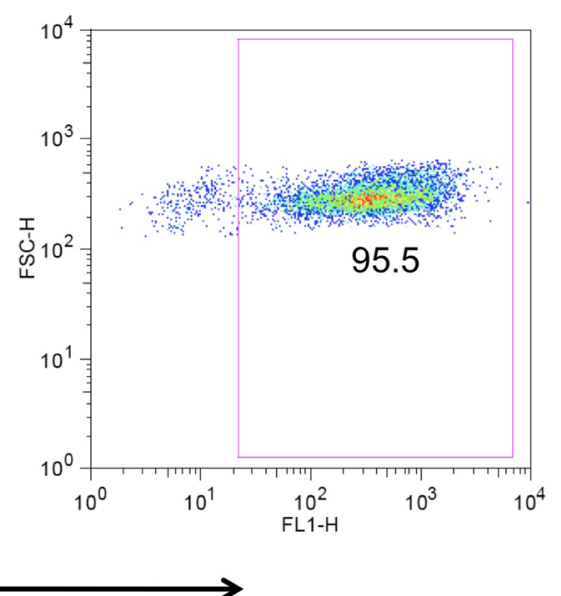

Fig. 2 Enrichment of cardiomyocytes with sodium L-lactate. a Summary of cardiac differentiation of different human iPS lines using efficient cardiac differentiation followed by lactate enrichment. b Flow cytometry analysis of cardiac-specific troponin $\mathrm{T}$ staining at day 16 of cardiac differentiation of line iLB-C1-30 m-r12 showed about $63 \%$ cTNT positive cardiomyocytes without lactate enrichment and $96 \%$ cTNT positive cells after lactate enrichment

structures (Suppl. Figure 1). They show different spatial orientation within the same cell as well as branching. Moreover, we observed sarcomer-like organization of contractile filaments (Fig. 4a). Additionally, fascia adherens-like and gapjunctions-like cellular contacts as well as initial sarcomeric organisation of actin and myosin filaments were detected. The sarcomeric structures of contractile filaments exhibited already identifiable A- and I-bands together with Z-lines as well as H-zones (Fig. 4b-d, Suppl. Figure 1).

\section{Discussion}

Our study reports a novel robust strategy to obtain cardiomyocytes from diverse human iPS lines that originate from a wide spectrum of state-of-the-art reprogramming 

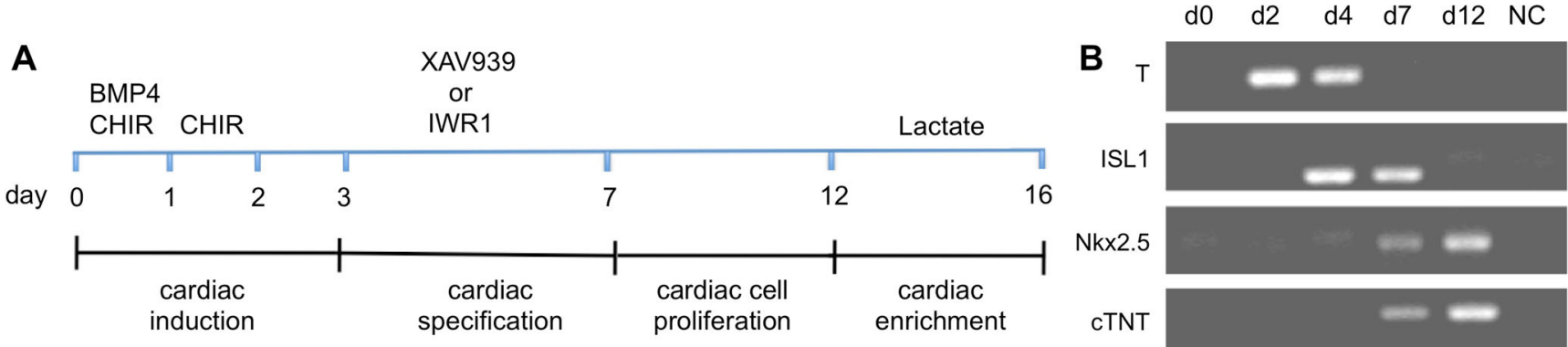

C
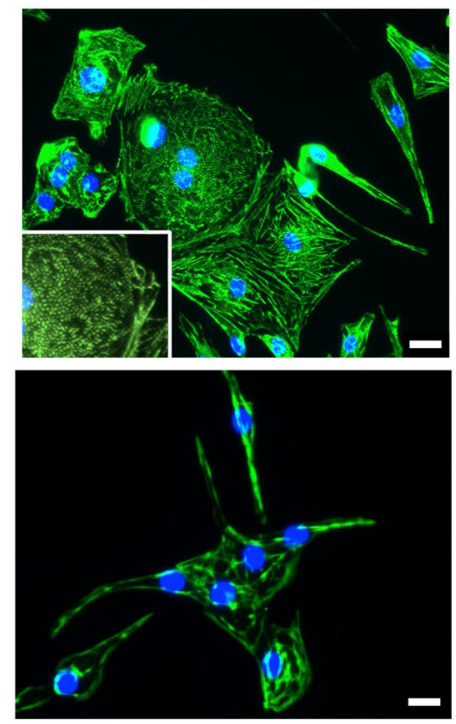

D

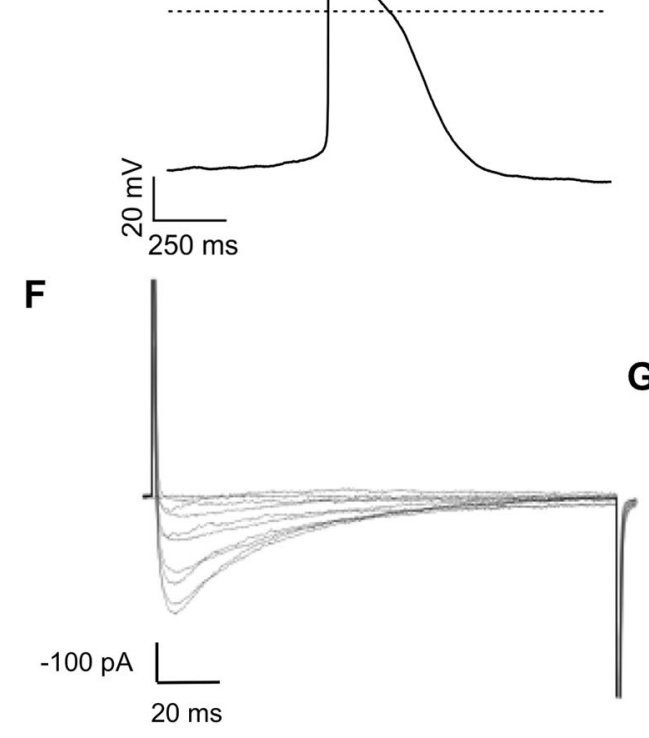

E

ISL1

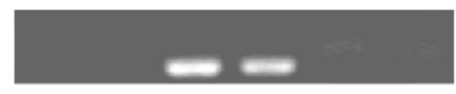

$\mathrm{Nk} \times 2.5$

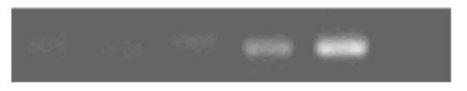

CTNT

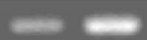

BA
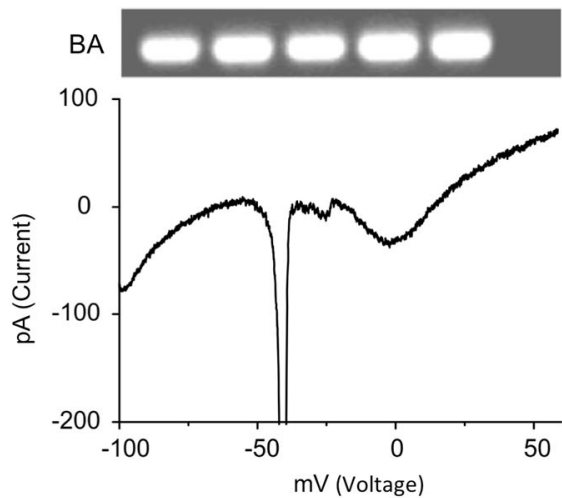

$\mathbf{G}$

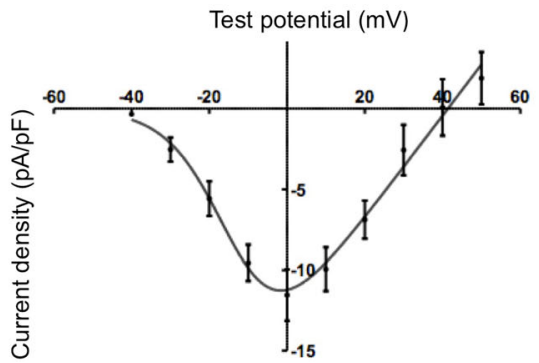

Fig. 3 Characterization of human iPSC (del-AR1034ZIMA 001) derived cardiomyocytes. a Scheme of efficient cardiac differentiation of human iPSC with combination of strong cardiac induction in early phase and cardiac enrichment in late phase. b RT-PCR analysis for mesendoderm, mesoderm, and cardiac specific gene expression $\mathbf{c}$ Immunohistochemical characterization of hiPS-derived cardiomyocytes using antibody against alpha-actinin (top) and cardiac troponin T (bottom). Scale bar: $40 \mu \mathrm{m}$. d Action potential recorded from a ventricular like cardiomyocyte. e
Typical activation of voltage dependent inward and outward currents following a ramp protocol in voltage clamp (-100 to $+60 \mathrm{mV}$ in $250 \mathrm{~ms})$. f Representative whole cell calcium current recording $(2 \mathrm{mM}$ extracellular $\mathrm{Ca}^{2+}$ ). Cells were depolarized from a holding potential of -80 to $-40 \mathrm{mV}$ for $45 \mathrm{~ms}$ in order to inactivate sodium channels. This prepulse was followed by test voltages ranging from -40 to $+50 \mathrm{mV}$ in $10 \mathrm{mV}$ steps (pulse duration $150 \mathrm{~ms}$ ). (G) Whole cell calcium current density-voltage relationship $(n=4)$ technology using an optimal combination of well-orchestrated extrinsic stimuli such as BMP4 and CHIR followed by WNT inhibition using XAV939 or IWR1 and enrichment of cardiomyocytes by supplying lactate as an energy source. The protocol described herein divides the whole differentiation process into three phases, namely cardiovascular induction, cardiac specification and cardiomyocyte enrichment. Our analysis revealed that efficient cardiac induction requires appropriate concentrations as well as precise timing of the right window of application of each chemical. We found out that sequential application of each chemical, BMP4 and CHIR, respectively, for up to two days is sufficient to drive the cells into cardiovascular fate which is consistent with earlier studies $[28,29,31,42]$. Notably, we have used basal media with insulin during first two days of induction phase. Previous studies have reported use of basal medium without insulin during cardiac differentiation due to its negative influence on cardiac specification of early mesoderm [28, 39]. However, our study shows that using basal medium without insulin from the beginning appears very stressful to the cells. Hence we decided to keep insulin for the initial two days and remove it during the specification phase in order to minimize cell death and its negative influence on cardiac specification. In order to achieve cardiac specification of early mesoderm, we used inhibitors of WNT signaling for up to 4 days as described in earlier studies $[28,29]$. We performed a comparative validation of molecules exhibiting WNT inhibitory activity using a WNT reporter cell line. It turned out that amongst four different compounds tested, XAV939 showed the strongest WNT inhibitory effect and was therefore used for all differentiation 
Fig. 4 Ultrastructural analysis of 21-day old human iPS (delAR1034ZIMA 001) cell-derived cardiomyocytes. a Two cells in close contact displaying sarcomer-like organization of contractile filaments. Scale bar: $1,000 \mathrm{~nm}(\mathbf{b}-\mathbf{c})$ Higher magnification showing the presence of fascia adherens-like and gap-junctions like cellular contacts and initial sarcomeric organisation of actin and myosin filaments. Scale bar: b 1,000 nm, c $250 \mathrm{~nm}$. d iPS cell-derived cardiomyocyte-like cells show sarcomer organization of contractile filaments with already identifiable A- and I-bands together with Z-lines as well as H-zones. Abbrevations: $m$ mitochondria, $N$ Nucleus, $F A$ - $I S$ Fascia adherens-like structure, GJ-lS Gap junctions-like structure, $Z \mathrm{Z}$-line, $\mathrm{H}$ H-zone. Scale Bar: $250 \mathrm{~nm}$
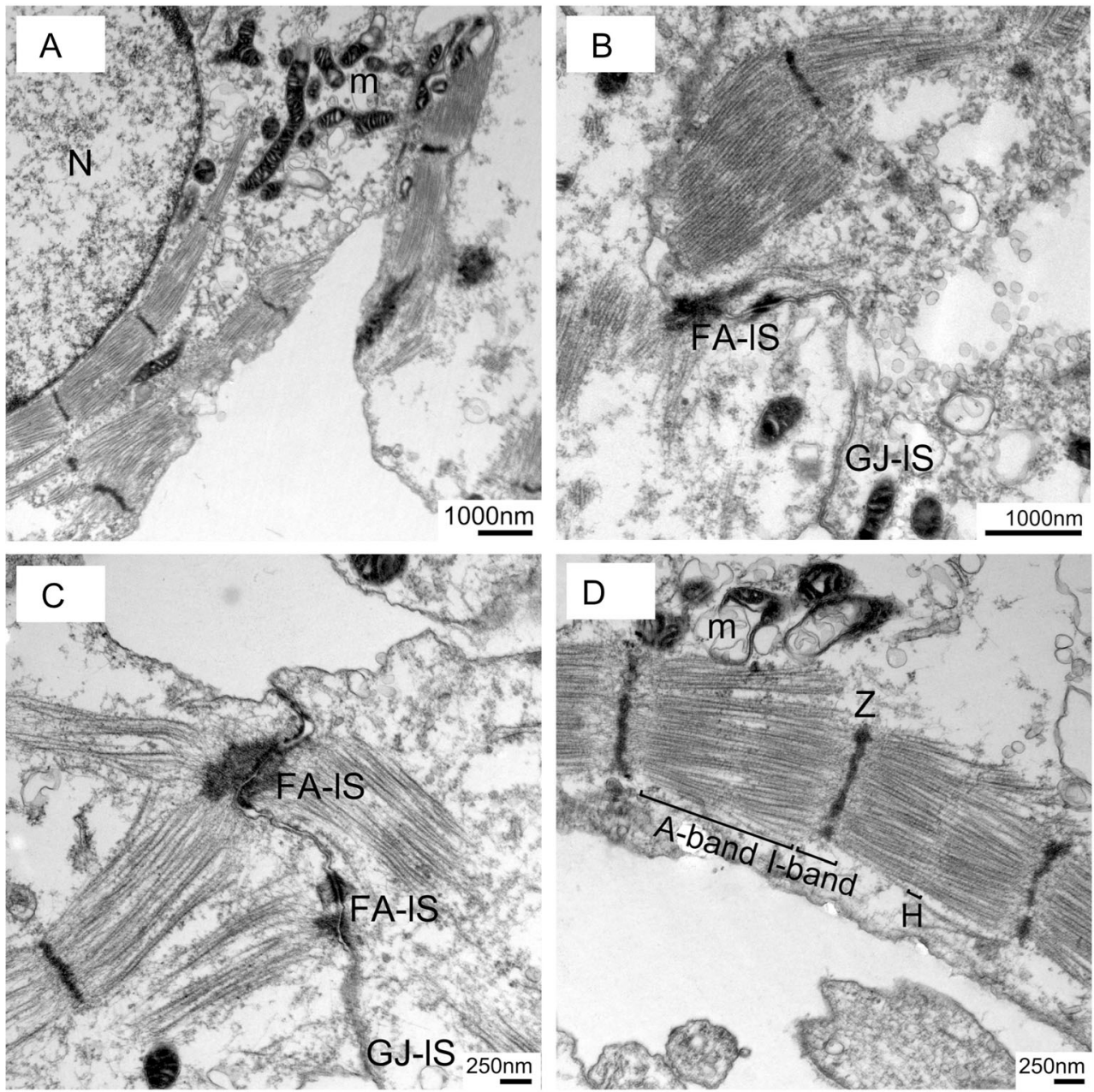

experiments. Moreover, we also achieved the same cardiac differentiation efficiency with previously described WNT inhibitor IWR-1 [27]. We observed different cardiac differentiation efficiencies in multiple iPS lines presumably due to the complexity of the signals, which is in accordance with recent studies $[17,18]$. We therefore elaborated further purification steps to improve the yield of cardiomyocytes with reduced line-to-line variability. We decided to assess the potential of lactate enrichment of cardiomyocytes and show a substantial cardiac enrichment of an iPS line (iLB-C1-30 m-r12) that exhibited relatively poor cardiomyocyte yield using our optimized chemical cocktail only. This indicates that the combination of extrinsically induced differentiation stimuli together with metabolic enrichment is an efficient means to overcome line-to-line variability of cardiomyocyte differentiation. There are various published studies showing successful cardiac differentiation of human pluripotent stem cells. However, as depicted in supplementary table 4 three key features separates our protocol from others i.e. i) sequential treatment of cardiac inducing factor with a clearly defined time window together with ii) an insulin switch, which in our experience is very critical to get reproducible results; iii) in addition our protocol includes a rapid and efficient way to enrich the cardiac population with lactate supplement. In order to evaluate the functional properties of cardiomyocytes, comprehensive validation was not only carried out at protein level by alpha-actinin and cTNT stainings but also by ultra-structural analysis. TEM results showed well organized sarcomeric structures in 21 day old cardiomyocytes with distinct I- and A-bands indicating relatively mature phenotype as described in earlier studies [15, 29]. Spontaneous action potentials of differentiated cells showed typical cardiomyocyte behaviour and we identified both ventricular and atrial-like shapes. Voltage ramps identified fast sodium, calcium as well as potassium currents. Characteristics of $\mathrm{Ca}^{2+}$ currents obtained from iPS-derived cardiomyocytes were similar to those recently obtained from murine ventricular myocytes [40]. I-V relationship furthermore perfectly agreed with data from HEK293 cells expressing recombinant human L-type $\mathrm{Ca}^{2+}$-channels suggesting that indeed iPS-derived cells express cardiac-like channel complexes consisting of pore-forming and auxiliary subunits [41]. Moreover calcium imaging further indicated usual calcium current activity seen in the case of human cardiomyocytes. In conclusion, cardiomyocytes obtained by 
our protocol have the potential for stem cell based therapies, drug toxicity as well as disease modeling studies. Hence we expect our protocol to provide a robust basis for scale-up production of functional iPS cell-derived cardiomyocytes.

Acknowledgments We would like to thank all members of the Stem Cell and Regenerative Medicine Group of the University of Wuerzburg for helpful suggestions and outstanding support. We also thank Johannes Jungverdorben from the University of Bonn, Leonhard Linta and Stefan Liebau from the University of Ulm, both Germany, for kindly providing us with human iPS lines. Frank Edenhofer was supported by grants from the Deutsche Forschungsgemeinschaft DFG (ED79/1-2), and the German Ministry of Education and Research, BMBF (01 GN 0813). Daniela Malan and Philipp Sasse were supported by the "StemCellFactory" project which is co-funded by the European Union (European Regional Development Fund - Investing in your future) and the German federal state North Rhine-Westphalia (NRW). Oliver Brüstle and Michael Peitz were supported by the 'StemCellFactory' projects (North Rhine Westphalian Programme for Regional Competitiveness and Employment (Bio.NRW); PtJ-Az.: z0911bt027i and North Rhine Westphalian Ministry of Innovation, Science and Research Programme "Translational Stem Cell Research" PtJ-Az.: z1403ts007e).

Disclosure of Potential Conflicts of Interest O.B. is co-founder and has stock in LIFE \& BRAIN GmbH, Bonn. The other authors declare no potential conflicts of interest.

Open Access This article is distributed under the terms of the Creative Commons Attribution License which permits any use, distribution, and reproduction in any medium, provided the original author(s) and the source are credited.

\section{References}

1. Lopez, A. D., Mathers, C. D., Ezzati, M., Jamison, D. T., \& Murray, C. J. (2006). Global and regional burden of disease and risk factors, 2001: systematic analysis of population health data. Lancet, 367, 1747-1757.

2. Orlic, D., Kajstura, J., Chimenti, S., et al. (2001). Mobilized bone marrow cells repair the infarcted heart, improving function and survival. Proceedings of the National Academy of Sciences of the United States of America, 98, 10344-10349.

3. Caplan, A. I., \& Dennis, J. E. (2006). Mesenchymal stem cells as trophic mediators. Journal of Cellular Biochemistry, 98, 1076-1084.

4. Zaruba, M. M., Soonpaa, M., Reuter, S., \& Field, L. J. (2010). Cardiomyogenic potential of C-kit(+)-expressing cells derived from neonatal and adult mouse hearts. Circulation, 121, 1992-2000.

5. Laugwitz, K. L., Moretti, A., Lam, J., et al. (2005). Postnatal ISL1+ cardioblasts enter fully differentiated cardiomyocyte lineages. Nature, 433, 647-653.

6. Choi, S. H., Jung, S. Y., Kwon, S. M., \& Baek, S. H. (2012). Perspectives on stem cell therapy for cardiac regeneration. Advances and challenges. Circulation Journal, 76, 1307-1312.

7. Cai, C. L., Liang, X., Shi, Y., et al. (2003). ISL1 identifies a cardiac progenitor population that proliferates prior to differentiation and contributes a majority of cells to the heart. Developmental Cell, 5, 877-889.

8. Hou, J., Wang, L., Jiang, J., et al. (2013). Cardiac stem cells and their roles in myocardial infarction. Stem Cell Reviews, 9, 326-338.
9. Murry, C. E., \& Keller, G. (2008). Differentiation of embryonic stem cells to clinically relevant populations: lessons from embryonic development. Cell, 132, 661-680.

10. Laflamme, M. A., \& Murry, C. E. (2011). Heart regeneration. Nature, 473, 326-335.

11. Takahashi, K., \& Yamanaka, S. (2006). Induction of pluripotent stem cells from mouse embryonic and adult fibroblast cultures by defined factors. Cell, 126, 663-676.

12. Passier, R., Oostwaard, D. W., Snapper, J., et al. (2005). Increased cardiomyocyte differentiation from human embryonic stem cells in serum-free cultures. Stem Cells, 23, 772-780.

13. Kehat, I., Kenyagin-Karsenti, D., Snir, M., et al. (2001). Human embryonic stem cells can differentiate into myocytes with structural and functional properties of cardiomyocytes. The Journal of Clinical Investigation, 108, 407-414.

14. Takei, S., Ichikawa, H., Johkura, K., et al. (2009). Bone morphogenetic protein-4 promotes induction of cardiomyocytes from human embryonic stem cells in serum-based embryoid body development. American Journal of Physiology. Heart and Circulatory Physiology, 296, H1793-H1803.

15. Mummery, C. L., Zhang, J., Ng, E. S., Elliott, D. A., Elefanty, A. G., \& Kamp, T. J. (2012). Differentiation of human embryonic stem cells and induced pluripotent stem cells to cardiomyocytes: a methods overview. Circulation Research, 111, 344-358.

16. Osafune, K., Caron, L., Borowiak, M., et al. (2008). Marked differences in differentiation propensity among human embryonic stem cell lines. Nature Biotechnology, 26, 313-315.

17. Kaichi, S., Hasegawa, K., Takaya, T., et al. (2010). Cell linedependent differentiation of induced pluripotent stem cells into cardiomyocytes in mice. Cardiovascular Research, 88, 314-323.

18. Ohno, Y., Yuasa, S., Egashira, T., et al. (2013). Distinct iPS Cells Show Different Cardiac Differentiation Efficiency. Stem Cells International, 2013, 659739.

19. Laflamme, M. A., Chen, K. Y., Naumova, A. V., et al. (2007). Cardiomyocytes derived from human embryonic stem cells in prosurvival factors enhance function of infarcted rat hearts. Nature Biotechnology, 25, 1015-1024.

20. Zhang, Q., Jiang, J., Han, P., et al. (2011). Direct differentiation of atrial and ventricular myocytes from human embryonic stem cells by alternating retinoid signals. Cell Research, 21, 579-587.

21. Uosaki, H., Fukushima, H., Takeuchi, A., et al. (2011). Efficient and scalable purification of cardiomyocytes from human embryonic and induced pluripotent stem cells by VCAM1 surface expression. PLoS One, 6, e23657.

22. Kattman, S. J., Witty, A. D., Gagliardi, M., et al. (2011). Stagespecific optimization of activin/nodal and BMP signaling promotes cardiac differentiation of mouse and human pluripotent stem cell lines. Cell Stem Cell, 8, 228-240.

23. Sa, S., \& McCloskey, K. E. (2012). Stage-specific cardiomyocyte differentiation method for $\mathrm{H} 7$ and $\mathrm{H} 9$ human embryonic stem cells. Stem Cell Reviews, 8, 1120-1128.

24. Burridge, P. W., Keller, G., Gold, J. D., \& Wu, J. C. (2012). Production of de novo cardiomyocytes: human pluripotent stem cell differentiation and direct reprogramming. Cell Stem Cell, 10, $16-28$.

25. Marvin, M. J., Di Rocco, G., Gardiner, A., Bush, S. M., \& Lassar, A. B. (2001). Inhibition of Wnt activity induces heart formation from posterior mesoderm. Genes and Development, 15, 316-327.

26. Ueno, S., Weidinger, G., Osugi, T., et al. (2007). Biphasic role for Wnt/beta-catenin signaling in cardiac specification in zebrafish and embryonic stem cells. Proceedings of the National Academy of Sciences of the United States of America, 104, 9685-9690.

27. Ren, Y., Lee, M. Y., Schliffke, S., et al. (2011). Small molecule Wnt inhibitors enhance the efficiency of BMP-4-directed cardiac differentiation of human pluripotent stem cells. Journal of Molecular and Cellular Cardiology, 51, 280-287. 
28. Lian, X., Hsiao, C., Wilson, G., et al. (2012). Robust cardiomyocyte differentiation from human pluripotent stem cells via temporal modulation of canonical Wnt signaling. Proceedings of the National Academy of Sciences of the United States of America, 109, E1848-E1857.

29. Minami, I., Yamada, K., Otsuji, T. G., et al. (2012). A small molecule that promotes cardiac differentiation of human pluripotent stem cells under defined, cytokine- and xeno-free conditions. Cell Reports, 2, 1448-1460.

30. Cao, N., Liu, Z., Chen, Z., et al. (2011). Ascorbic acid enhances the cardiac differentiation of induced pluripotent stem cells through promoting the proliferation of cardiac progenitor cells. Cell Research, 22, 219-236.

31. Cao, N., Liang, H., Huang, J., et al. (2013). Highly efficient induction and long-term maintenance of multipotent cardiovascular progenitors from human pluripotent stem cells under defined conditions. Cell Research, 23, 1119-1132.

32. Tohyama, S., Hattori, F., Sano, M., et al. (2013). Distinct metabolic flow enables large-scale purification of mouse and human pluripotent stem cell-derived cardiomyocytes. Cell Stem Cell, 12, 127-137.

33. Kadari, A., Lu, M., Li, M., et al. (2014). Excision of viral reprogramming cassettes by Cre protein transduction enables rapid, robust and efficient derivation of transgene-free human induced pluripotent stem cells. Stem Cell Research \& Therapy, 5, 47.

34. Linta, L., Stockmann, M., Kleinhans, K. N., et al. (2012). Rat embryonic fibroblasts improve reprogramming of human keratinocytes into induced pluripotent stem cells. Stem Cells and Development, 21, 965-976.

35. Koch, P., Breuer, P., Peitz, M., et al. (2011). Excitation-induced ataxin-3 aggregation in neurons from patients with MachadoJoseph disease. Nature, 480, 543-546.
36. Fuerer, C., \& Nusse, R. (2010). Lentiviral vectors to probe and manipulate the Wnt signaling pathway. PloS One, 5, e9370.

37. Yang, L., Soonpaa, M. H., Adler, E. D., et al. (2008). Human cardiovascular progenitor cells develop from a KDR+embryonicstem-cell-derived population. Nature, 453, 524-528.

38. Malan, D., Friedrichs, S., Fleischmann, B. K., \& Sasse, P. (2011). Cardiomyocytes obtained from induced pluripotent stem cells with long-QT syndrome 3 recapitulate typical disease-specific features in vitro. Circulation Research, 109, 841-847.

39. Lian, X., Zhang, J., Zhu, K., Kamp, T. J., \& Palecek, S. P. (2013). Insulin inhibits cardiac mesoderm, not mesendoderm, formation during cardiac differentiation of human pluripotent stem cells and modulation of canonical Wnt signaling can rescue this inhibition. Stem Cells, 31, 447-457.

40. Beetz, N., Hein, L., Meszaros, J., et al. (2009). Transgenic simulation of human heart failure-like L-type $\mathrm{Ca} 2+$-channels: implications for fibrosis and heart rate in mice. Cardiovascular Research, 84, 396- 406.

41. Jangsangthong, W., Kuzmenkina, E., Khan, I. F., Matthes, J., Hullin, R., \& Herzig, S. (2010). Inactivation of L-type calcium channels is determined by the length of the $\mathrm{N}$ terminus of mutant beta(1) subunits. Pflügers Archiv, 459, 399-411.

42. Carpenter, L., Carr, C., Yang, C. T., Stuckey, D. J., Clarke, K., \& Watt, S. M. (2011). Efficient differentiation of human induced pluripotent stem cells generates cardiac cells that provide protection following myocardial infarction in the rat. Stem Cells and Development, 21, 977-986.

43. Dambrot, C., Buermans, HP., Varga, E., et al. (2014). Strategies for rapidly mapping proviral integration sites and assessing cardiogenic potential of nascent human induced pluripotent stem cell clones. Exp Cell Res. 